\title{
On the Origin of Morphospecies Using Bayesian Phylogenetics to Quantify the Mode of Ancestor Descendant Relationships in Paleozoic Invertebrates
}

\author{
David W Bapst, \\ Selina Cole, David Wright, Melanie Hopkins, \\ Michael Melchin, Curtis Congreve, James Lamsdell, \\ Charles E. Mitchell, Sandra Carlson
}




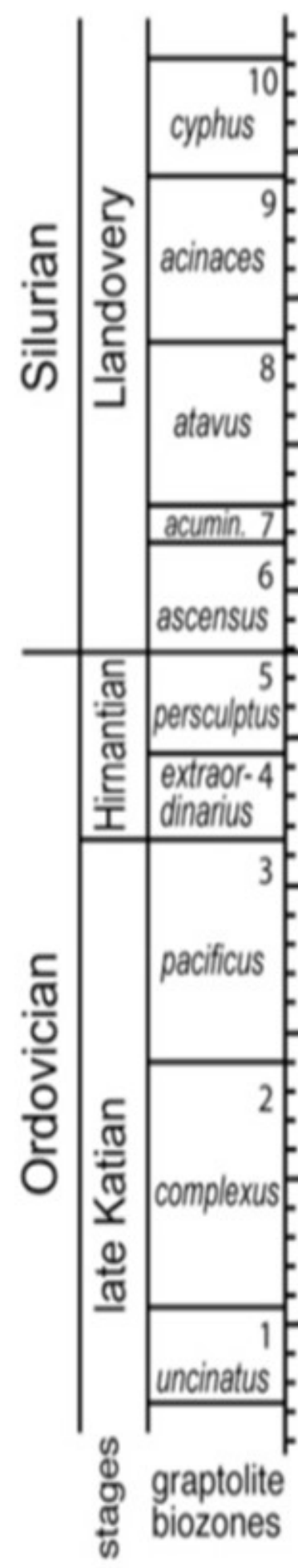

\section{Morphotaxa in \\ the Fossil Record}

- Often, we find specimens with similar morphology, but from different localities and stratigraphic height

- We use those features to define morphotaxa that persist over geologic time 


\section{A New Era of Ancestors on Trees}

- We expect to sample ancestors (especially indirect ancestors) from first principles

Foote, 1996
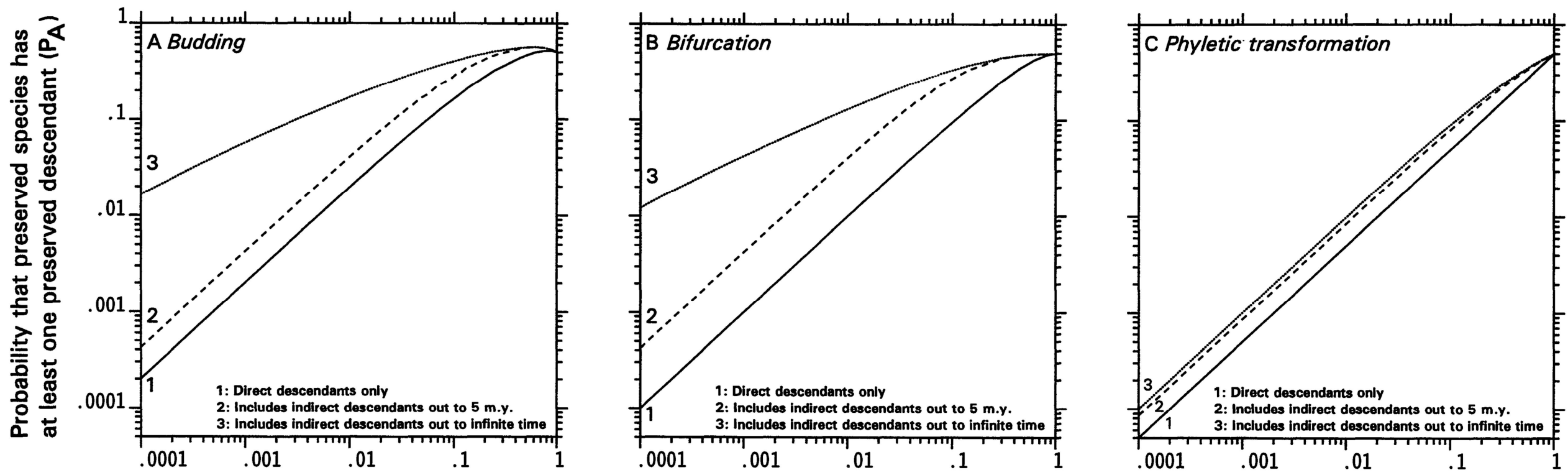

Proportion of species preserved $\left(P_{P}\right)$ 


\section{A New Era of Ancestors on Trees}

- We expect to sample ancestors (especially indirect ancestors) from first principles

- Bayesian tip-dating and other methods let us quantify support for ancestor-descendant relationships

- Different methods seem to assign similar support

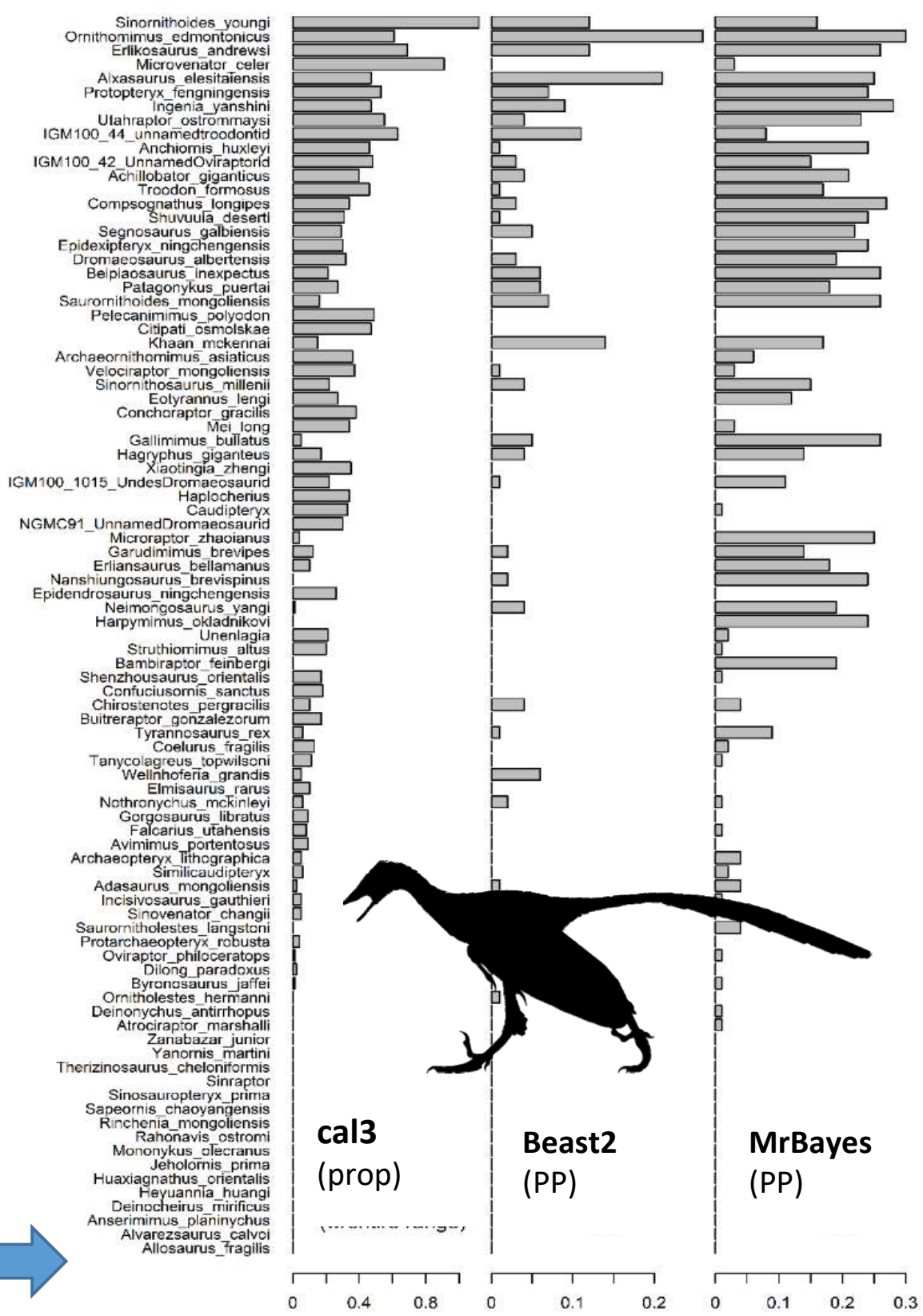


How often do we infer sampled ancestors in real fossil records? Which mode of differentiation is most common among persistent morphotaxa?

- Implications for our understanding of speciation, trait evolution, extent that taxonomic turnover could be inflated by pseudo-extinction

- Apply tip-dating to morphological matrices from the well-sampled marine invertebrate record

- More than 35 pre-existing datasets; 26 examined today

- Predominantly Paleozoic groups, such as brachiopods, graptolites, trilobites, crinoids, other echinoderms

- Varying matrix size, taxonomic level (species vs genera), sampling schema (e.g. clade-focused vs interval focused) 
Sampled-Ancestor Tip-Dating with the Fossilized Birth-Death Model in MrBayes

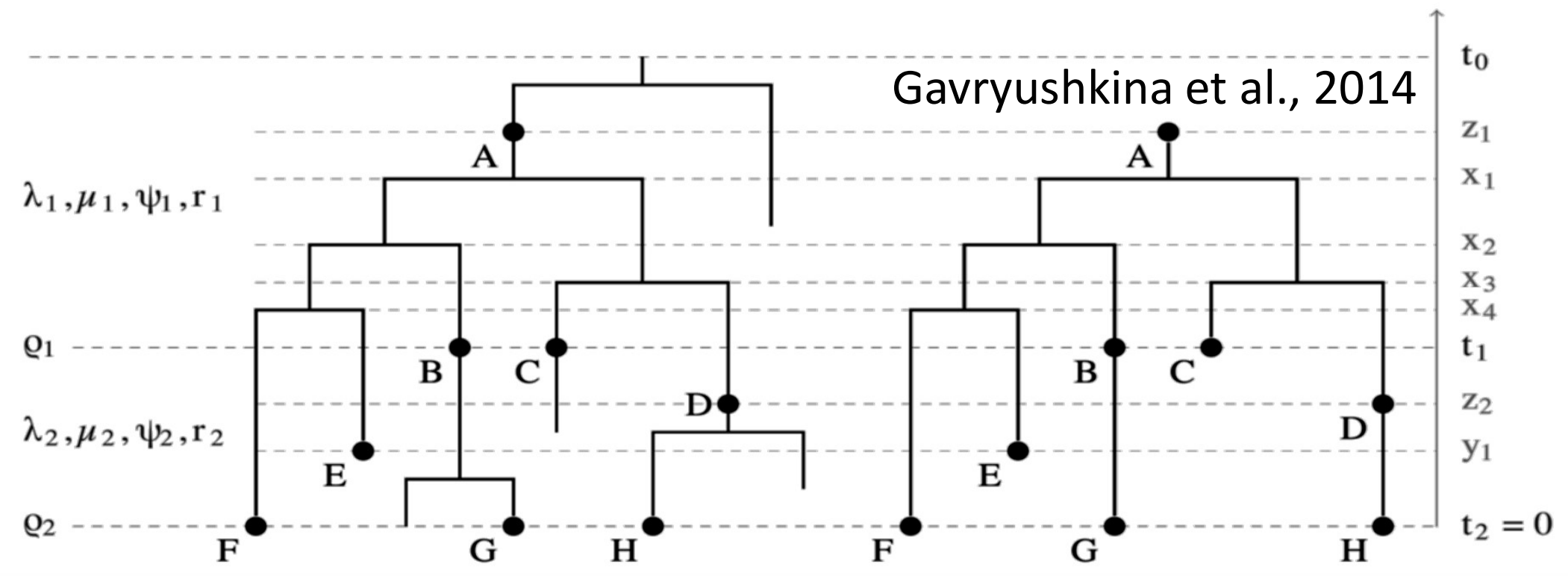

- Currently available tip-dating methods limited to considering taxon units as point occurrences in time

- To consider relationships among persistent morphotaxa, we treat first \& last appearances as two separate taxon units, with identical morphological characters but different time of appearance

- Functions for automating this are in $\mathrm{R}$ package paleotree 


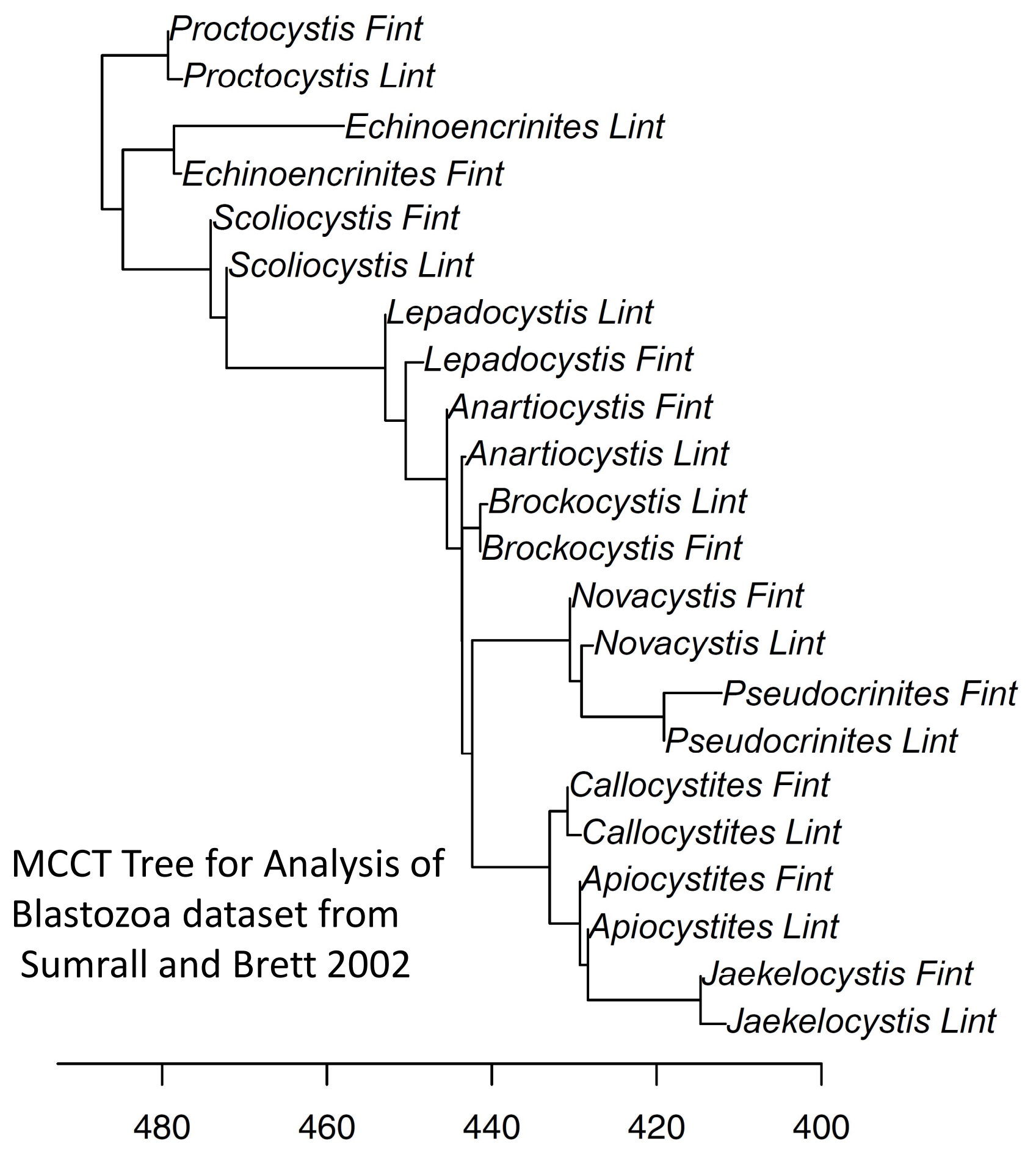




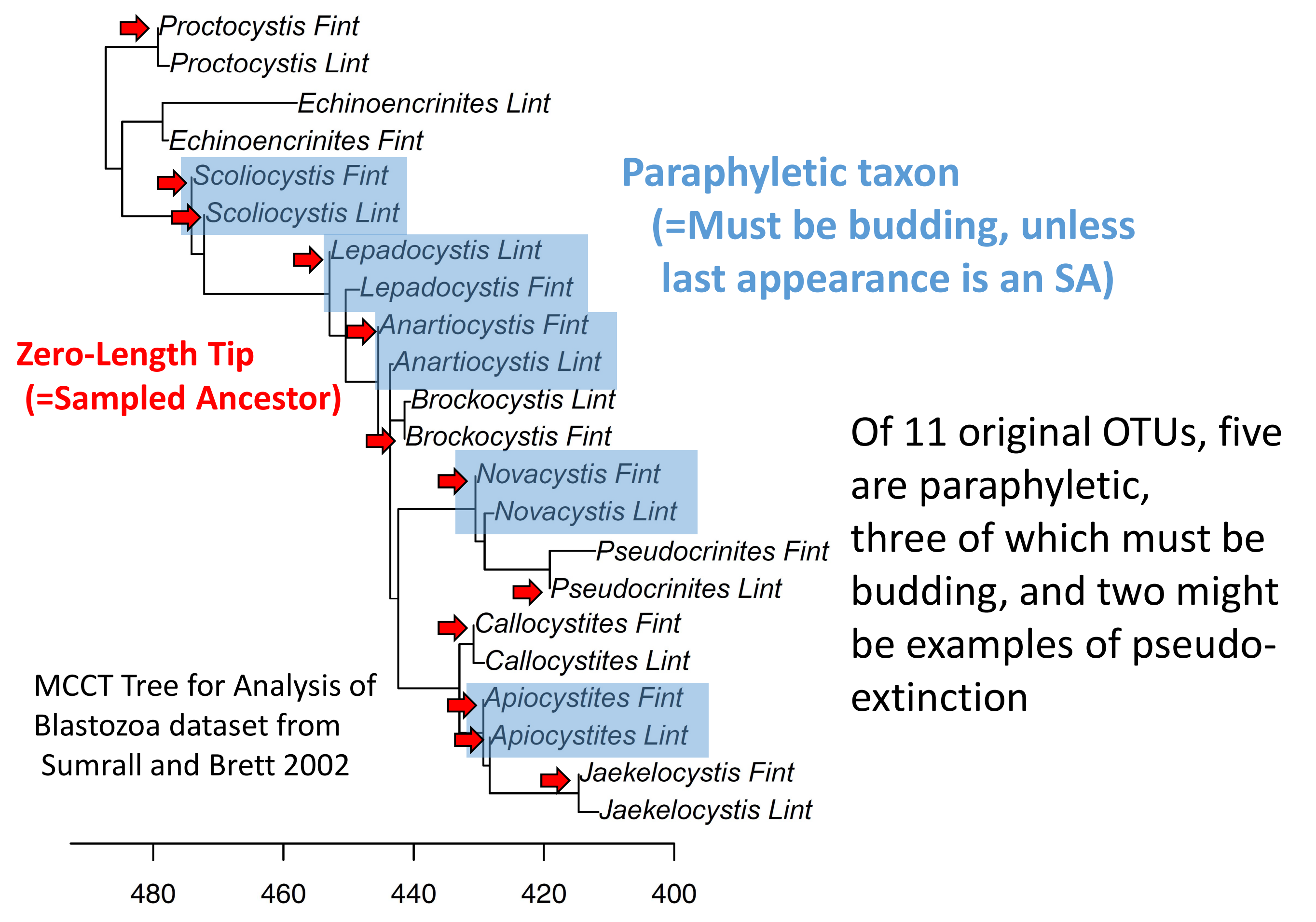


$\Rightarrow$ Proctocystis Fint

Proctocystis Lint

Echinoencrinites Lint

Echinoencrinites Fint

$\Rightarrow$ Scoliocystis Fint

$\Rightarrow$ Scoliocystis Lint

$\Rightarrow$ Lepadocystis Lint

Lepadocystis Fint

\section{Paraphyletic taxon}

(=Must be budding, unless last appearance is an SA)

$\rightarrow$ Anartiocvstis Fint

\section{But this is just a single tree pulled}

from a very large posterior - need to

measure and summarize these

patterns across the posteriors

Sumrall and Brett 2002

$\Rightarrow$ Jaekelocystis Fint

\begin{tabular}{cccccc}
\hline 1 & 1 & & & \\
480 & 460 & 440 & 420 & 400
\end{tabular}




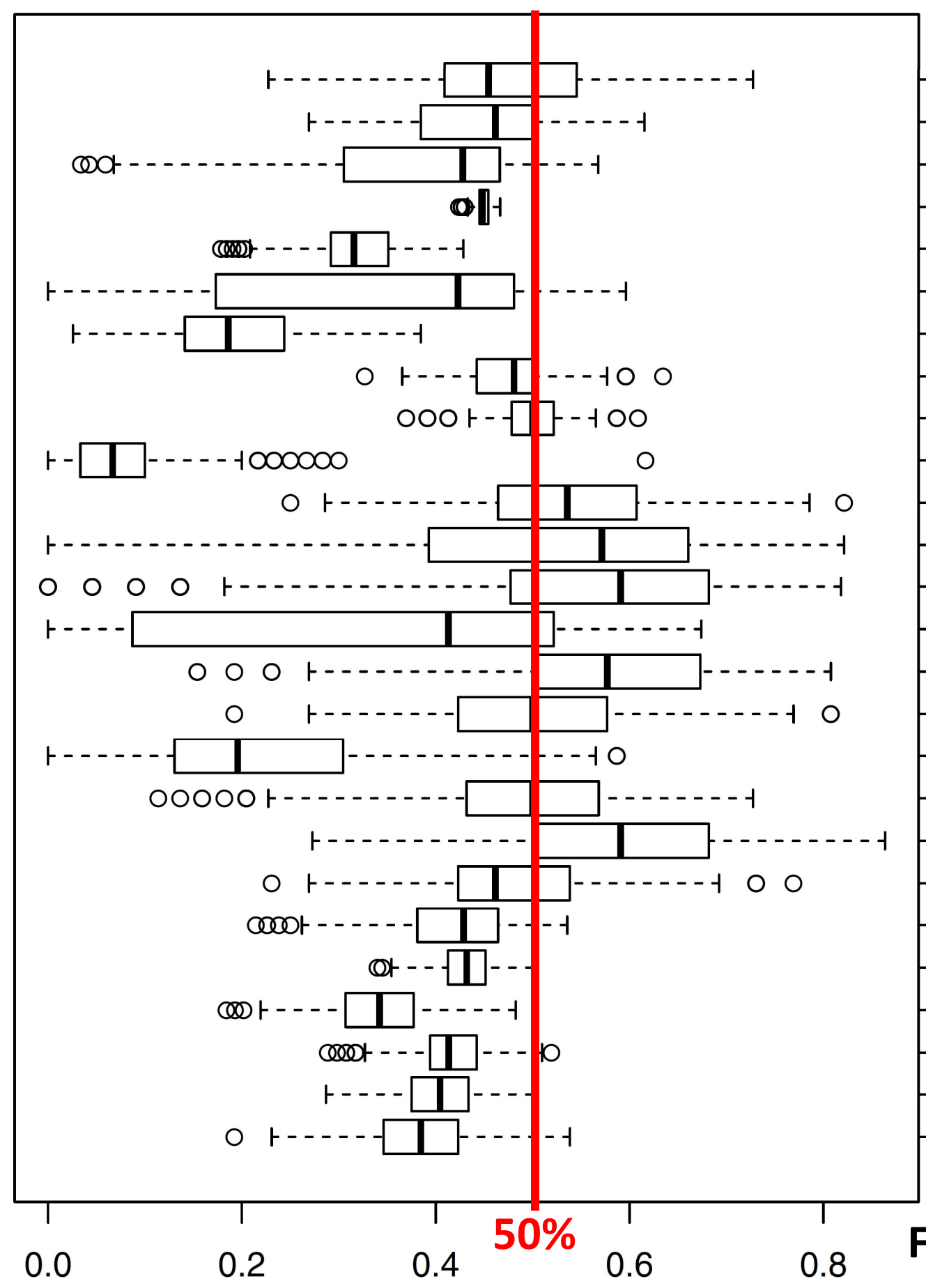

-36_Hebertella

- 35_Plaesiomys

- 31_Neograpta

- 30_Echinoids

- 24_Pentamerides

- 23_Stenoscismatoids

- 22_DevonianTerebratulides

- 21_Orthids

- 20_Stylophora

- 19_Stylophora

- 18_Pelmatozoans

- 17_Crinoidea

- 16_Crinoidea

- 15_Crinoidea

- 14_Crinoidea

- 13_Crinoidea

- 12_Crinoidea

- 11_Cincta

- 10_Blastozoa

- 9_Blastozoa

- 7_Pterochephaliidae

- 6_Diplobathrids

- 5_Disparids

- 4_Camerates

- 2_Blastozoa

- 1_Balnibarbiinae

First \& last appearances are separate OTUs, thus might expect $50 \%$ to be Proportion of Tip OTUs Placed as Sampled Ancestors placed as sampled ancestors 


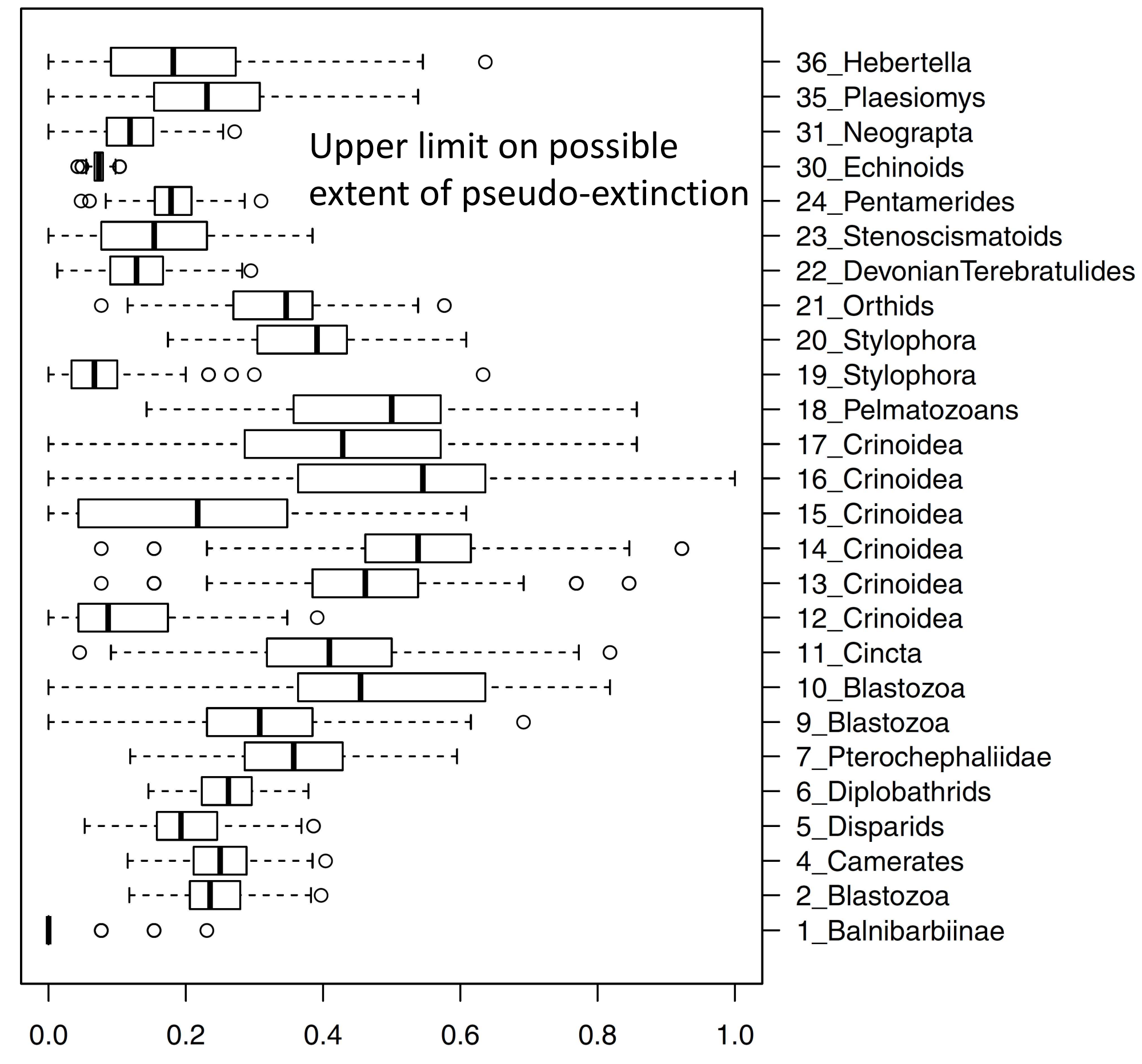

Proportion of Last Occurrences Placed as Sampled Ancestors 


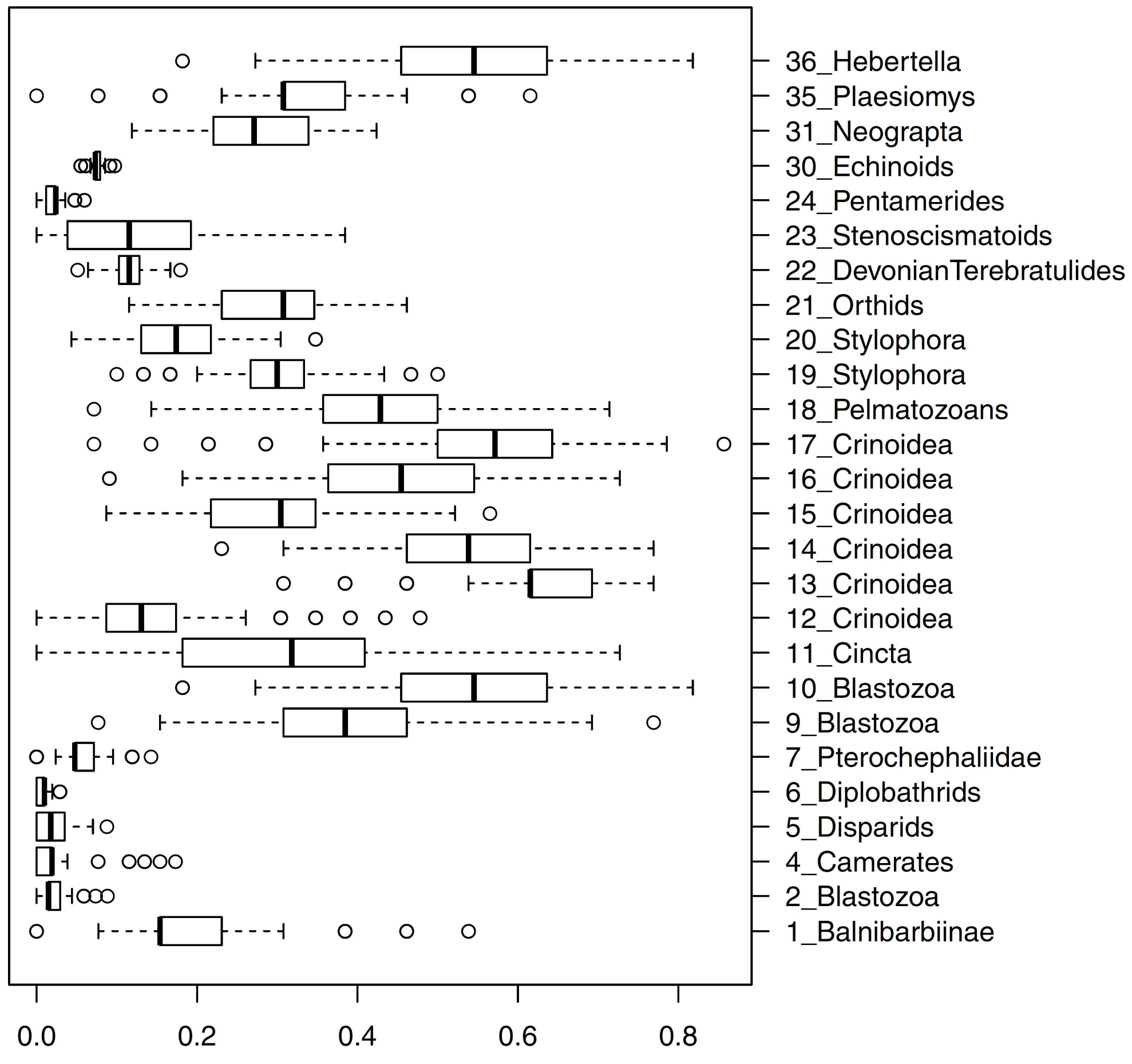

Paraphyletic Proportion of First-Last Pairs 
The Pattern of

Ancestor-Descendant Relationships

- Treating fossil morphotaxa as more than single OTUs sheds light on patterns of ancestor-descendant relationships

- Ancestor-descendant relationships considerably across different datasets

- Effects of taxonomic practices? Sampling? Biological differences?

Thanks for listening! Questions? 


Sigmocheilus pogonipensis-

Sigmocheilus flabellifer

Pterocephalia concavaPterocephalia sanctisabae

Housia ovataHousia vacuna

Parahousia subequalisParahousia constricta

Aphelaspis subditusDicanthopyge quadrata

Aphelaspis hagueiAphelaspis subditus

Strigambitus transversusStrigambitus utahensis

Sigmocheilus nothaSigmocheilus pogonipensis

Tumicephalus depressusHousia ovata

Cernuolimbus laevifronsCernuolimbus depressus

Dicanthopyge quadrataDicanthopyge convergens

Prehousia indentaPrehousia alata

Dicanthopyge convergensDicanthopyge reductus

Aphelaspis longispinaTumicephalus depressus

Dicanthopyge quadrataDicanthopyge reductus

Strigambitus utahensisStrigambitus transversus

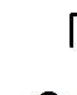
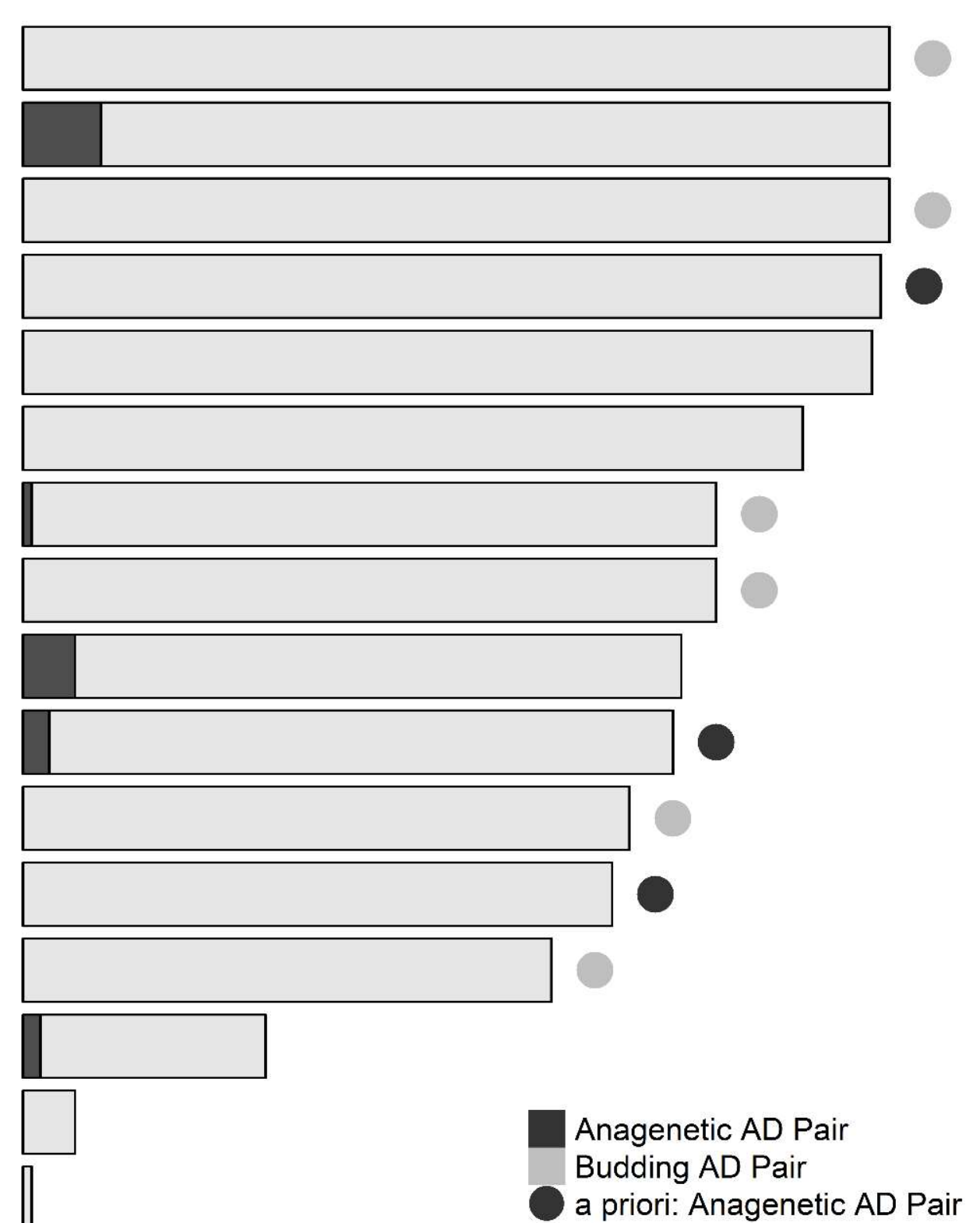

Anagenetic AD Pair Budding AD Pair

a priori: Anagenetic AD Pair a priori: Budding AD Pair

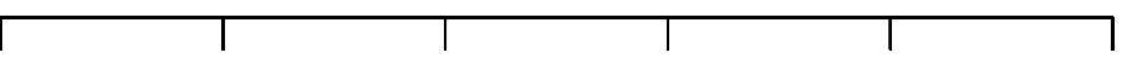

0.2
0.4

0.6

0.8

1.0
$>$ Each pair is a stacked barplot

$>$ Dots indicate putative pairs

$>$ Evidence for all a priori AD pairs, \& a few extra

$>$ cal3 finds very little support for anagenesis

$>$ Given biases, perhaps entirely budding?

Proportion of cal3 Runs Placed as Ancestor-Descendant Pair

Bapst \& Hopkins 2017 



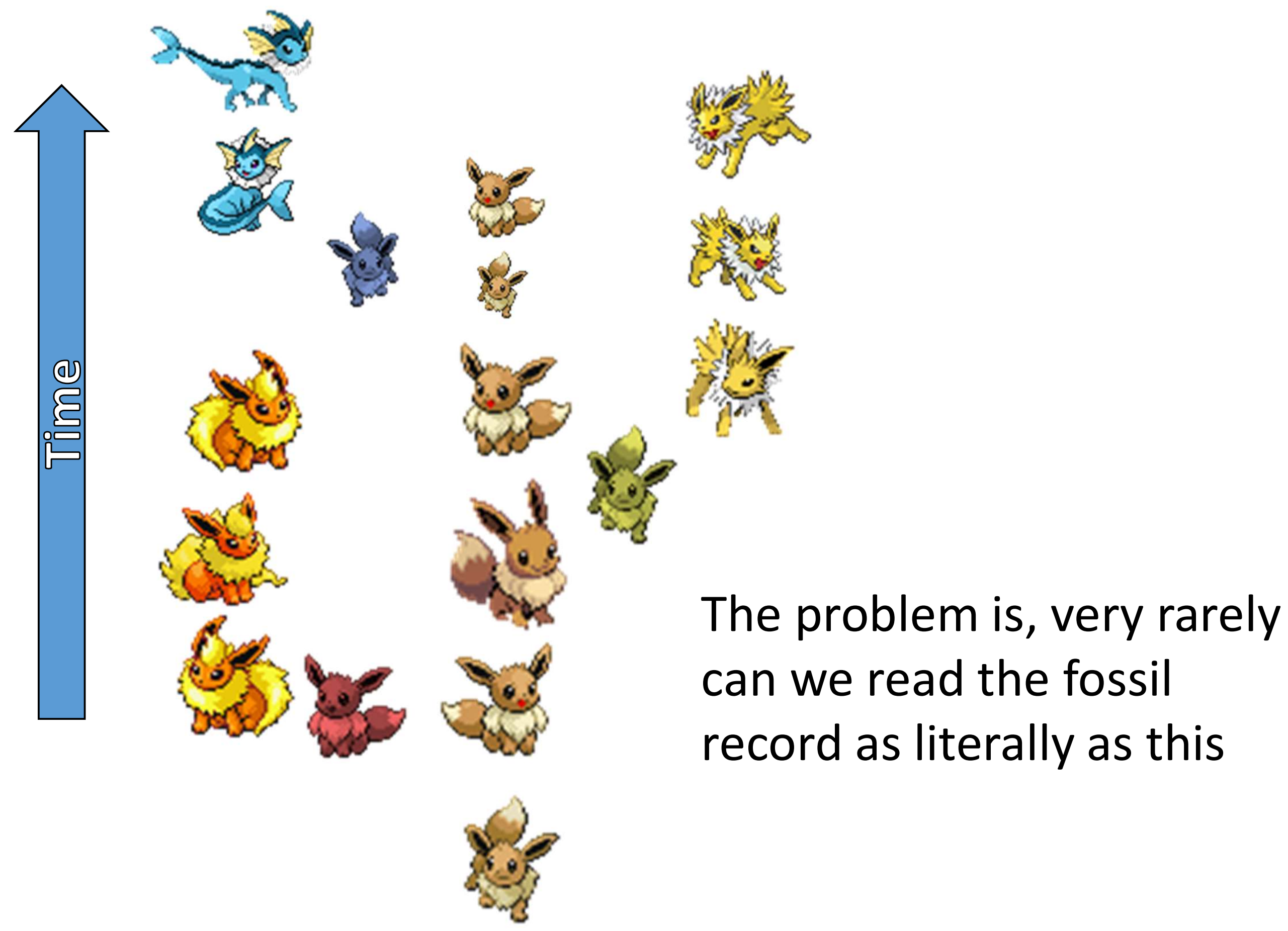




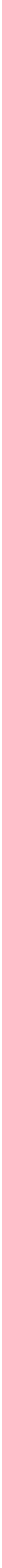




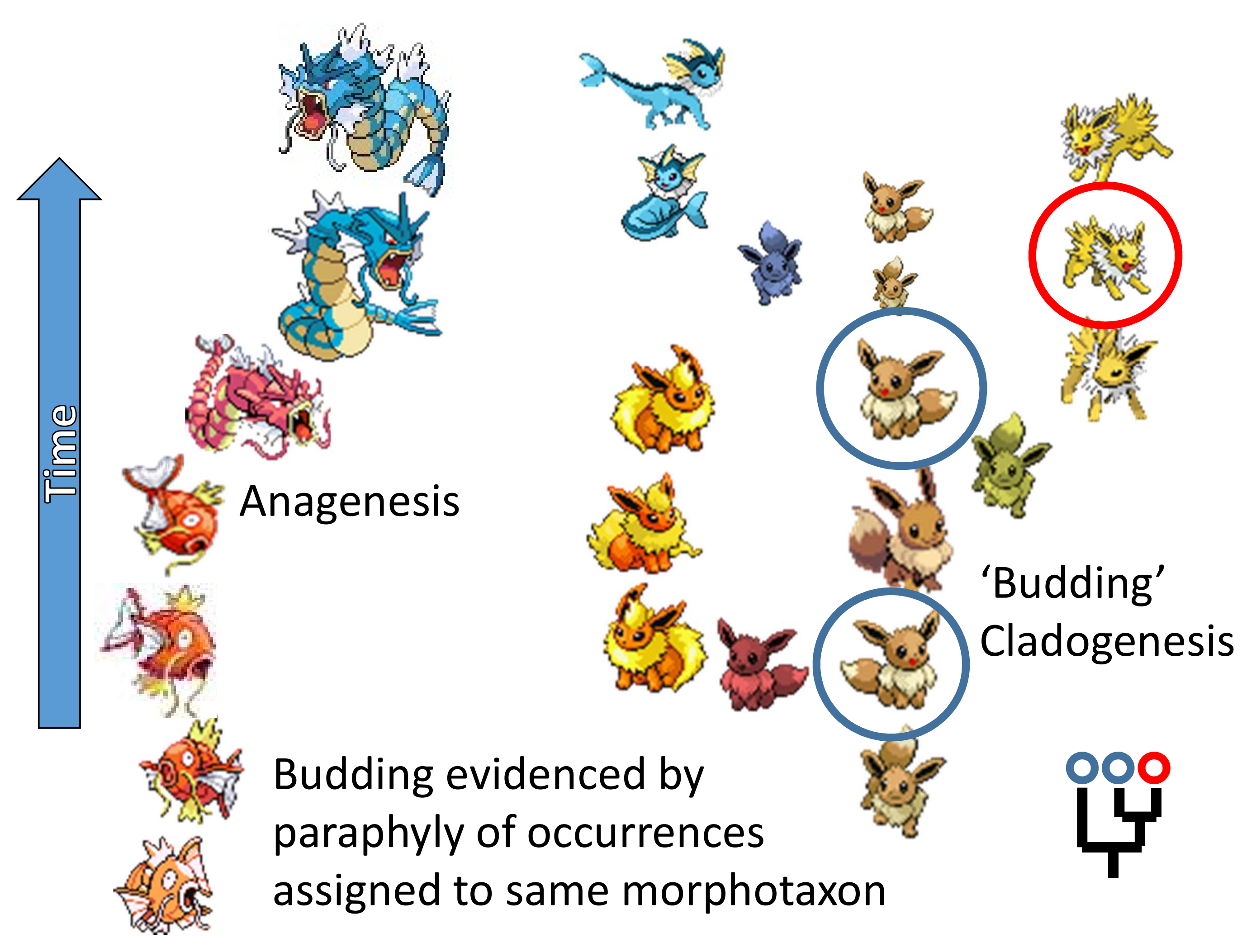




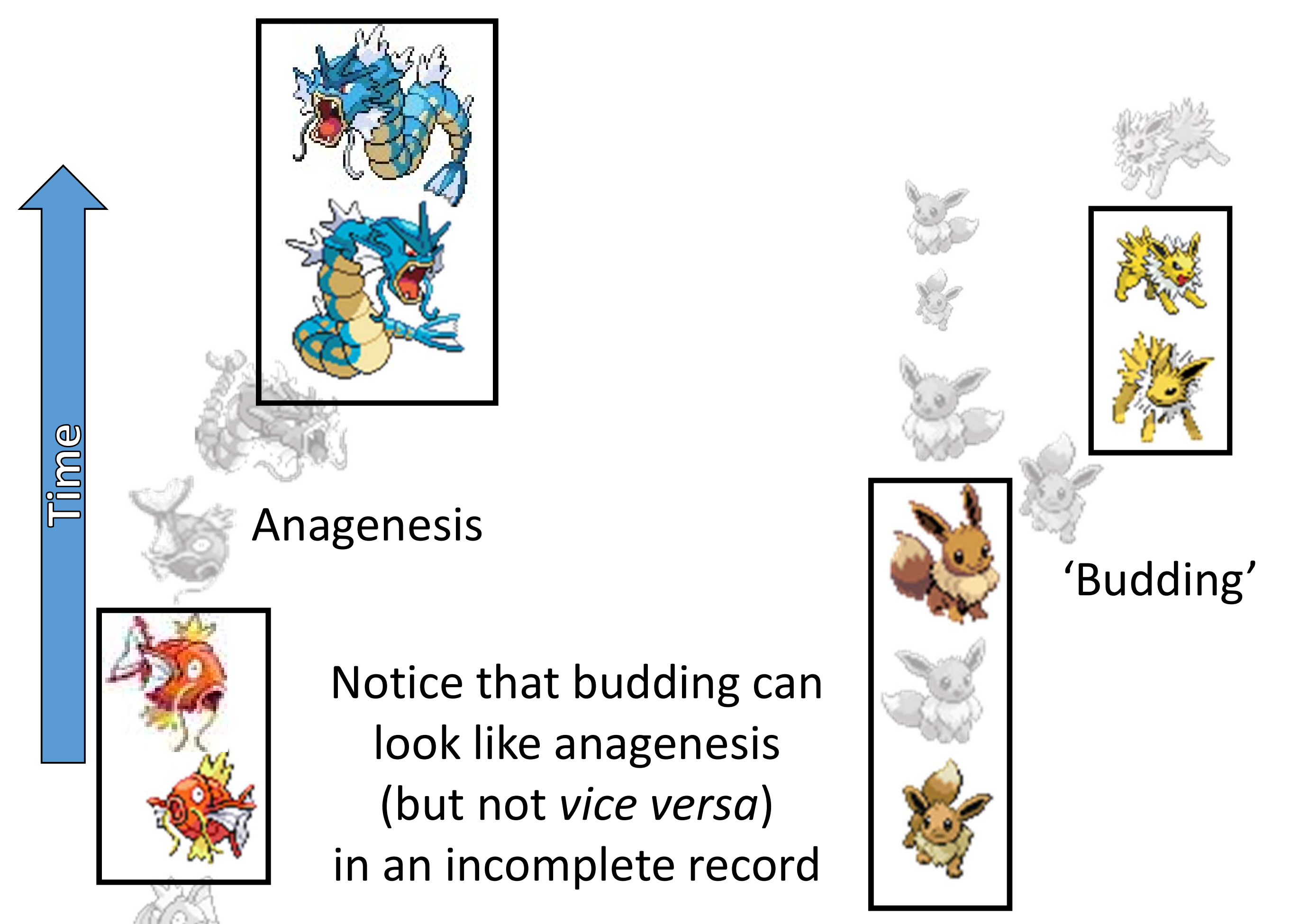




\section{Gaps in Densely-Sampled Fossil Records}

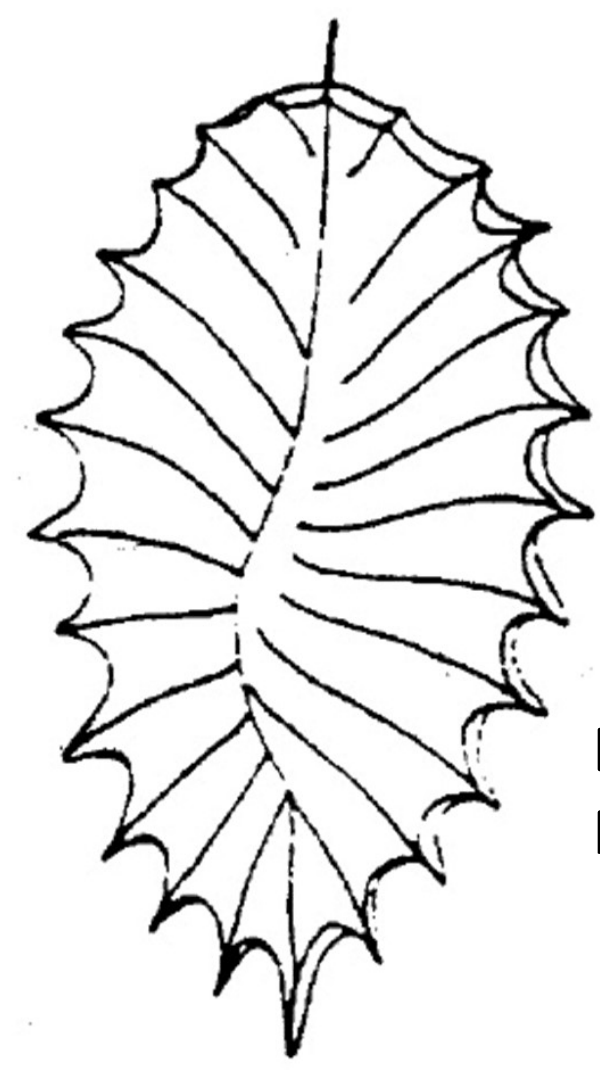

Sinoretiograptus Latest Katian

Bergstromgraptus Middle Darrwillian

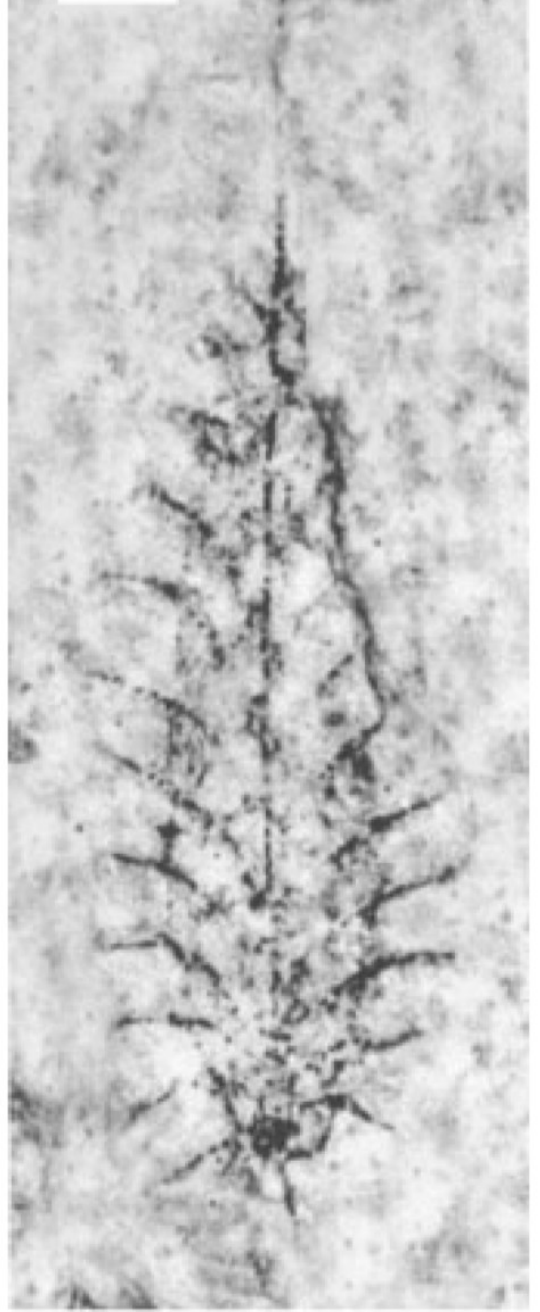

- Closest relatives separated by a 15 to 20 million year gap in this lineage:

- Were the intermediates living somewhere else? Open ocean?

Maletz and Zhang, 2003; Vandenberg, 2003; C.E. Mitchell 


\section{In The Age Of Ancestor Inference...}

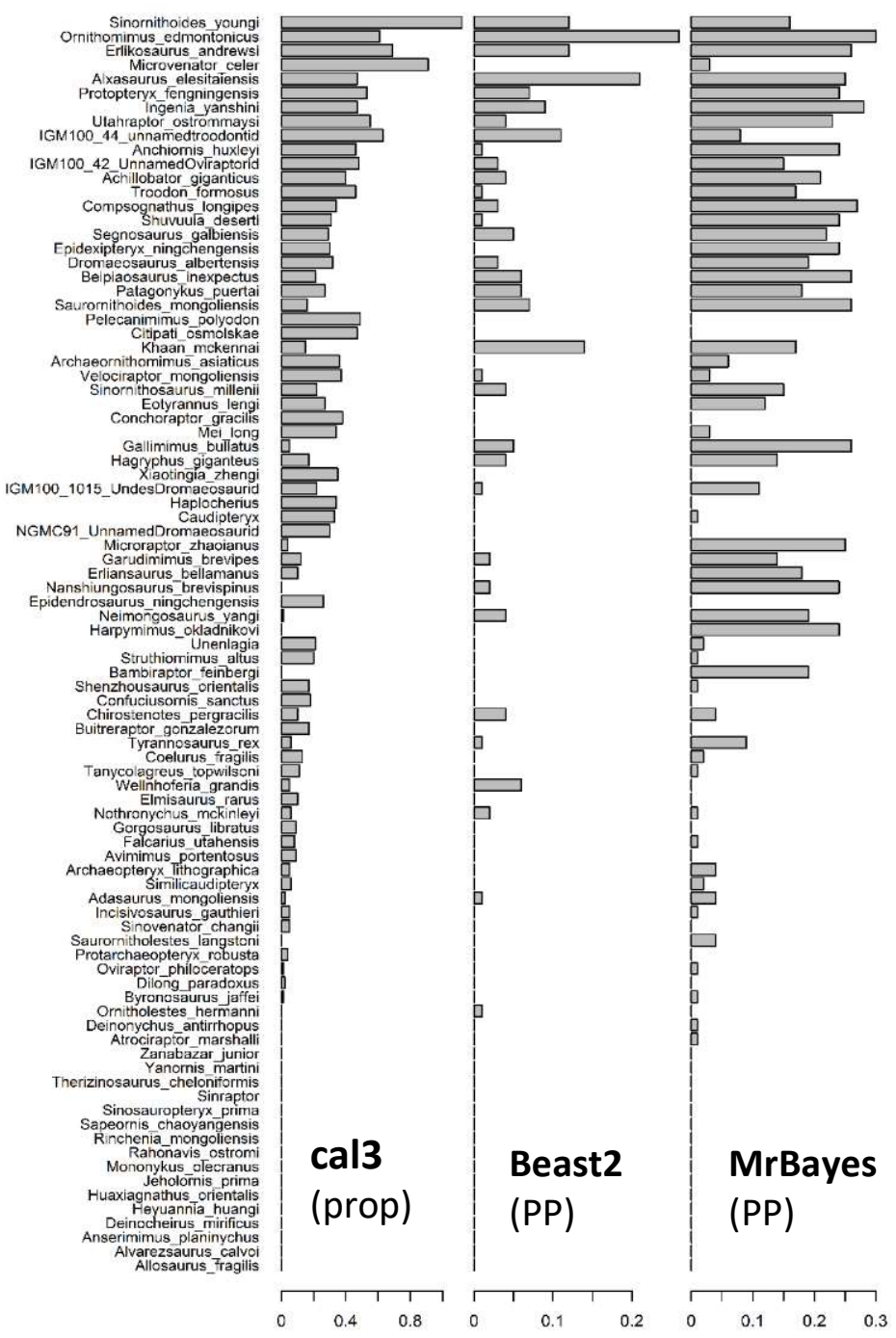

(cal3 is an off-brand tip-dating lite)

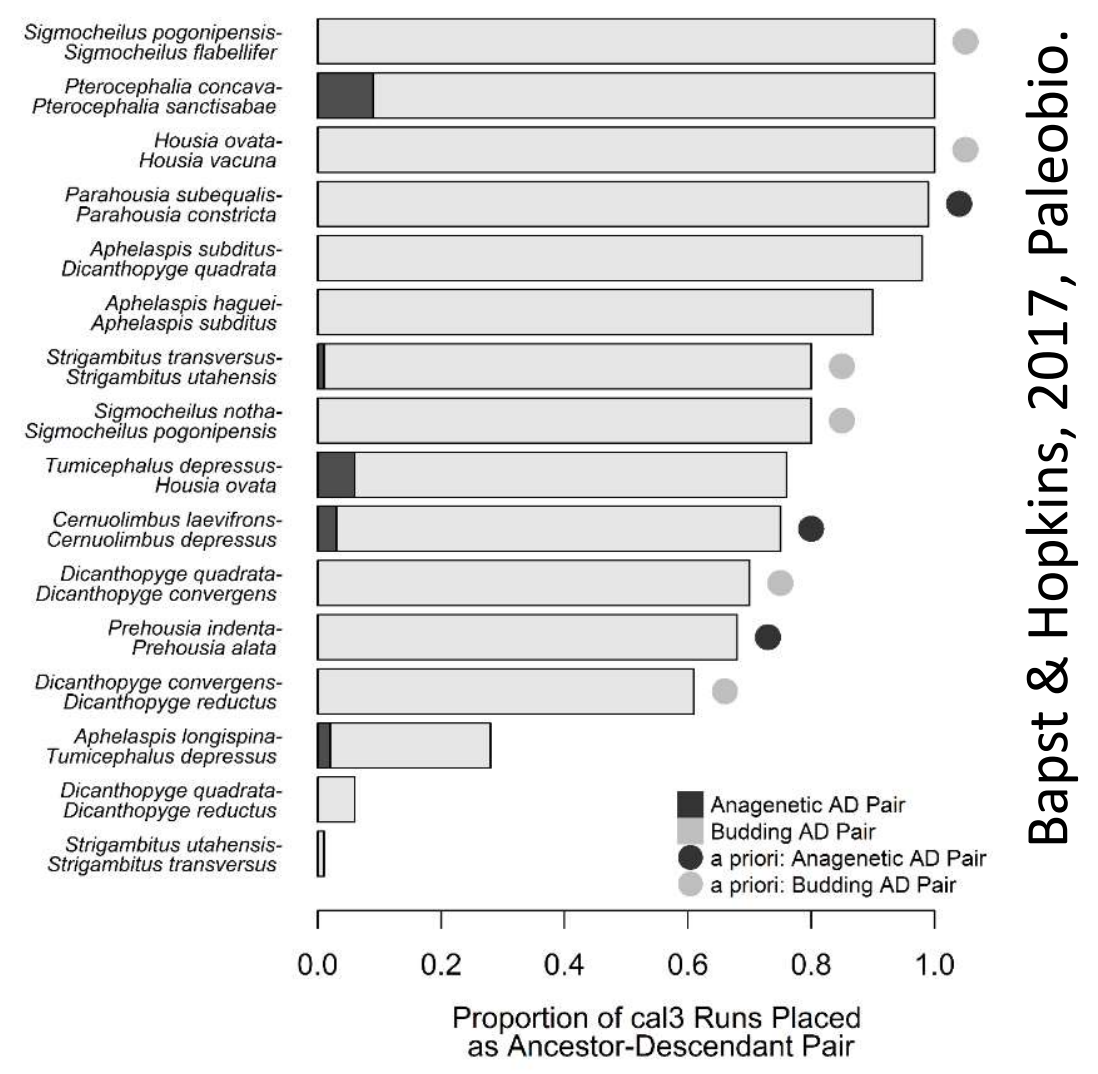

- Different methods agree on placing ancestors [dinosaurs]

- Quantitative inferences agree with previous putative pairs of ancestor-descendants [trilobites] 